ISSN 0103-5150

Fisioter. Mov., Curitiba, v. 25, n. 3, p. 525-532, jul./set. 2012 Licenciado sob uma Licença Creative Commons

\title{
Influência do posicionamento dos membros superiores sobre parâmetros ventilatórios em indivíduos adultos
}

\author{
Influence of the upper limbs' position on \\ ventilatory parameters in adults subjects
}

\begin{abstract}
Akinori Cardozo Nagato ${ }^{[a]}$, Claudia Regina Carvalho Barboza ${ }^{[b]}$, Rafaela Gontijo Manso ${ }^{[c]}$, Maria de Fátima Ferreira de Oliveira ${ }^{[\mathrm{d}]}$, Marco Aurélio dos Santos Silva ${ }^{[\mathrm{e}]}$, Frank Silva Bezerra ${ }^{[\mathrm{f}]}$
\end{abstract}

[a] Fisioterapeuta, mestre em Biologia Humana e Experimental (UERJ), professor assistente II do curso de Fisioterapia, Laboratório de Biomorfologia e Patologia Experimental (LBPE) da Universidade Severino Sombra (USS), Vassouras, RJ Brasil, e-mail: akynory@hotmail.com

[b] Fisioterapeuta, graduada na Universidade Severino Sombra (USS), Vassouras, RJ - Brasil, e-mail: claudiarcbarboza@yahoo.com.br

[c] Acadêmica do curso de Medicina da Universidade Federal de Ouro Preto (UFOP), Ouro Preto, MG - Brasil, e-mail: rafaela.bh89@hotmail.com

[d] Fisioterapeuta, professora assistente do curso de Fisioterapia, Hospital Universitário Sul Fluminense (HUSF), Universidade Severino Sombra (USS), Vassouras, RJ - Brasil, e-mail: mariaffdeoliveira@gmail.com

[e] Fisioterapeuta, mestre em Biologia Humana e Experimental pela Universidade Estadual do Rio de Janeiro (UERJ), professor assistente II do curso de Fisioterapia, Laboratório de Biomorfologia e Patologia Experimental (LBPE) da Universidade Severino Sombra (USS), Vassouras, RJ - Brasil, e-mail: marcoaurelioanato@hotmail.com

[f] Fisioterapeuta, doutor em Ciências Morfológicas pela Universidade Federal do Rio de Janeiro (UFRJ), professor adjunto II do Departamento de Ciências Biológicas (DECBI), Laboratório de Bioquímica Metabólica da Universidade Federal de Ouro Preto (UFOP), Ouro Preto, MG - Brasil, e-mail: frank@iceb.ufop.br

\section{Resumo}

Introdução: A ventilação pulmonar depende da integridade da parede torácica. 0 posicionamento da parede torácica e/ou as disfunções dos músculos ventilatórios diminuem os volumes e as capacidades pulmonares, o fluxo de ar nas vias aéreas e as pressões inspiratória e expiratória máximas. 0 posicionamento dos membros superiores interfere na dinâmica da caixa torácica. Objetivos: Verificar o efeito do posicionamento dos membros superiores com o ombro a $90^{\circ}$ de abdução e rotação externa, associado à flexão de cotovelo a $90^{\circ}$ sobre a função ventilatória pulmonar. Métodos: Os indivíduos foram submetidos ao Questionário 
Respiratório sugerido pela American Thoracic Society - Division of Lung Diseases (ATS-DLD) e à ficha individual de pesquisa, para avaliação clínica da função ventilatória pulmonar. Resultados: Foi observado que o volume minuto e o volume corrente nos indivíduos saudáveis no pós-posicionamento aumentaram quando comparados à situação de pré-posicionamento, de $9,46 \pm 1,15 \mathrm{~L} / \mathrm{min}$. para 11,89 $\pm 1,54 \mathrm{~L} / \mathrm{min}$. e de 565,8 $\pm 83,84 \mathrm{ml}$ para $752,4 \pm 105,5 \mathrm{ml}$, respectivamente. Conclusão: 0 posicionamento dos membros pode ser priorizado antes das técnicas reexpansivas pulmonares. Programas de reabilitação pulmonar, de maneira geral, podem incluir o posicionamento correto dos membros superiores, pois isso coloca em vantagem os músculos que atuam sobre a caixa torácica, melhorando o desempenho da ventilação pulmonar.

Palavras-chave: Ventilação pulmonar. Posicionamento dos membros superiores. Função pulmonar.

\section{Abstract}

Introduction: Pulmonary ventilation depends on the integrity of the chest wall. The positioning of the chest wall and/or the disorders of ventilatory muscles decrease the lung volume and capacity, the flow of airway pressure and inspiratory and expiratory pressures. The positioning of the upper limbs interferes with the dynamics of the chest. Objectives: To determine the effect of positioning of the arms with the shoulder $90^{\circ}$ abduction and external rotation associated with elbow flexion to $90^{\circ}$ on the pulmonary ventilatory function. Methods: The subjects underwent Respiratory Questionnaire suggested by the American Thoracic Society - Division of Lung Diseases (ATS-DLD) and the individual records to search for clinical assessment of ventilatory lung function.

Results: We found out that the minute volume and tidal volume in healthy subjects in post-position increased when compared to the situation of pre-positioning from $9.46 \pm 1.15 \mathrm{~L} / \mathrm{min}$. to $11.89 \pm 1.54 \mathrm{~L} / \mathrm{min}$. and from $565.8 \pm 83.84 \mathrm{ml}$ to $752.4 \pm 105.5 \mathrm{~mL}$, respectively. Conclusion: The placement of members can be prioritized before lung reexpansion techniques. Pulmonary rehabilitation programs in general may include the correct positioning of the upper limbs, as this represents an advantage to muscles that act on the rib cage, improving the performance of ventilation.

Keywords: Pulmonary ventilation. Position of the upper limbs. Pulmonary function.

\section{Introdução}

A principal função do sistema respiratório é a hematose, mecanismo que proporciona o transporte de $\mathrm{O}_{2}$ para todos os tecidos e a remoção de $\mathrm{CO}_{2}$ resultante da respiração celular. A bomba ventilatória, constituída pela caixa torácica e pelo abdome, move-se durante o ciclo ventilatório, promovendo mudanças na geometria da parede do tórax e gerando alterações no volume e na pressão intratorácica, os quais possibilitam o empuxo de fluxo de ar para insuflar e desinsuflar os pulmões alternadamente. Já os músculos ventilatórios atuam sob controle voluntário e involuntário e se contraem em intervalos regulares, de modo a manter a função ventilatória em níveis satisfatórios (1).

A função muscular ventilatória pode ser afetada, em maior ou menor proporção, por fatores como idade, gênero, peso, altura e fatores genéticos, bem como doenças pulmonares, neuromusculares e alterações estruturais da caixa torácica. Na presença de doença pulmonar ou alteração da complacência da parede torácica, observa-se alteração dos volumes e das capacidades pulmonares e anormalidade da função muscular respiratória $(2,3)$.

A força gerada pelos músculos respiratórios pode ser avaliada pela pressão por eles gerada, e a velocidade de contração ou estiramento, pelo fluxo gerado, ao passo que o estiramento muscular é estimado pela alteração do volume deslocado. Entre os recursos utilizados para avaliar qualitativa e quantitativamente a função ventilatória pulmonar, são utilizados: (a) espirômetro, que mensura as alterações dos volumes e as capacidades pulmonares, como capacidade vital forçada (CVF), soma do volume de reserva inspiratória (VRI), do volume corrente (VC) e do volume de reserva expiratória (VRE); (b) manovacuômetro, que mede a força dos músculos ventilatórios através da medida da pressão inspiratória máxima (PI máx) e da pressão expiratória máxima (PE máx); (c) exame 
de pico de fluxo expiratório (PFE), que mede o fluxo máximo após expiração forçada; e (d) ventilômetro, que mensura o volume minuto (VM), volume total de ar inalado e exalado em um minuto, estando o paciente em repouso, produto do VC e da frequência respiratória (FR). Esses testes contribuem, portanto, para o esclarecimento da limitação ventilatória pulmonar, para a identificação da natureza fisiopatológica dos distúrbios ventilatórios, de sintomas e da tolerância do indivíduo aos procedimentos terapêuticos, bem como no acompanhamento da evolução e da avaliação da resposta terapêutica (4-8).

Na prática clínica, o aumento da expansibilidade torácica, em virtude do posicionamento correto dos MMSS, é acompanhado do subjetivo relato do paciente em relação a um conforto ventilatório. Logo, o objetivo do presente estudo foi verificar o efeito do posicionamento dos MMSS com o ombro a $90^{\circ}$ de abdução e rotação externa, associado à flexão de cotovelo a $90^{\circ}$ sobre a função ventilatória pulmonar. Para isso, foi medido o volume minuto, o volume corrente através da ventilometria, a capacidade vital forçada, o pico de fluxo expiratório, a pressão expiratória máxima e a pressão inspiratória máxima.

\section{Materiais e métodos}

0 estudo foi realizado no primeiro semestre letivo de 2009, na Fundação Educacional Severino Sombra, da cidade de Vassouras (RJ). Participaram do estudo 16 indivíduos adultos do sexo masculino: 12 indivíduos saudáveis do quadro de funcionários em atividade do Hospital Universitário Sul-Fluminense (HUSF) e da Universidade Severino Sombra (USS), cadastrados e acompanhados regularmente pelo Setor de Medicina do Trabalho dessa instituição, os quais se disponibilizaram voluntariamente após convite do pesquisador, e quatro indivíduos hospitalizados da enfermaria masculina do HUSF, clinicamente estáveis.

Os 16 indivíduos estudados foram divididos em dois grupos: (a) grupo de indivíduos saudáveis $(\mathrm{S})$ e (b) grupo de indivíduos hospitalizados $(\mathrm{H})$, com tempo médio de internação hospitalar de 7,5 dias. Os indivíduos foram submetidos ao Questionário Respiratório sugerido pela American Thoracic Society - Division of Lung Diseases (ATS-DLD) e à ficha individual de pesquisa para avaliação clínica e avaliação da função ventilatória pulmonar.
Na avaliação clínica, realizou-se anamnese, constando dados de identificação pessoal - iniciais do nome e idade. Obtiveram-se dados antropométricos - peso e altura -, por meio da balança mecânica antropométrica para adultos (Welmy, Brasil), e dados clínicos - história da doença atual (HDA), história patológica pregressa (HPP), história social (HS), coleta dos sinais vitais, com o indivíduo sentado à beira do leito, pressão arterial sistêmica (PAS) (esfignomanômetro aneróide - Solidor, China e estetoscópio - Solidor, China); frequência cardíaca (FC), através de palpação da artéria radial; frequência respiratória (FR), através da visualização das incursões respiratórias durante um minuto e de análise do padrão ventilatório.

A avaliação da função ventilatória pulmonar foi realizada em duas etapas. A primeira etapa foi denominada pré-posicionamento, na qual os indivíduos foram posicionados em decúbito dorsal, com a cabeceira da maca elevada em posição Fowler a

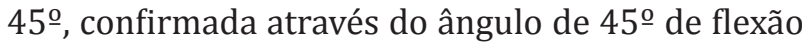
da coluna lombar com goniômetro (Carci, Brasil) e com reposicionamento da cintura escapular. Os MMSS mantiveram-se estendidos ao longo do tronco, com as palmas das mãos voltadas para cima e com os joelhos em semiflexão (Figura 1).

Nesse posicionamento inicial foram realizados perimetria torácica com fita antropométrica (Sanny Medical, Brasil) e ventilometria, no período de um minuto (ventilômetro - Ferraris Mark 8, England), por meio da solicitação da ventilação em repouso, com mensuração simultânea da FR, a fim de se estimar

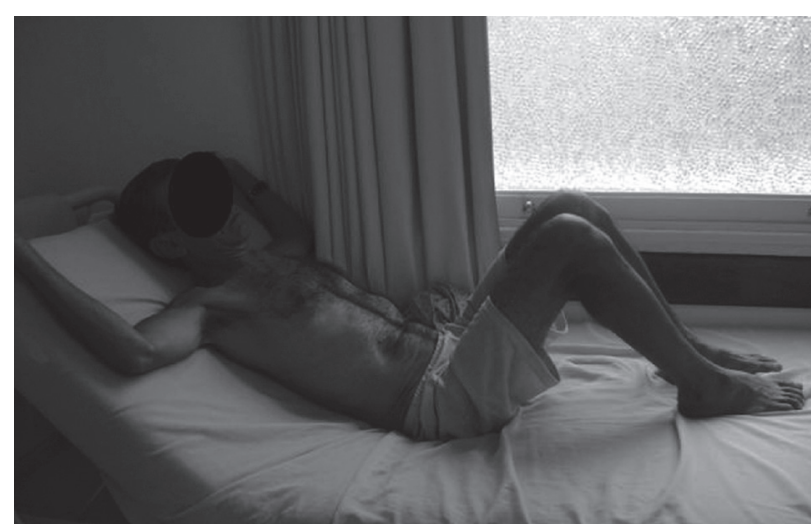

Figura 1 - Posicionamento em decúbito dorsal com a cabeceira da maca elevada em posição Fowler a $45^{\circ}$, MMSS posicionados em abdução e rotação externa de ombro a $90^{\circ}$, associado à flexão de cotovelo a $90^{\circ}$ 
o VC. Mediu-se o PFE (Peak Flow Meter - Assess, Estados Unidos) através de inspiração profunda seguida de expiração rápida. Realizou-se, ainda, espirometria (espirômetro portátil - Graham-Field, United States) através de uma inspiração máxima seguida de expiração máxima prolongada, com a finalidade de se medir CVF e manovacuometria (manovacuômetro Marshall Town, United States), pela solicitação de inspiração profunda seguida de expiração vigorosa, para mensuração da PE máx, e uma inspiração vigorosa, contra o manômetro, para a mensuração da PI máx.

Na segunda etapa, denominada pós-posicionamento, os MMSS foram posicionados em abdução e rotação externa de ombro a $90^{\circ}$, associados à flexão de cotovelo a $90^{\circ}$. Os indivíduos mantiveram-se nesse posicionamento durante dez minutos, e as mesmas medidas foram realizadas para a verificação da interferência desse posicionamento na ventilação pulmonar.

As técnicas de ventilometria, espirometria e manovacuometria foram realizadas com o auxílio de um clipe nasal. Para medida de PFE, CVF, PE máx e PI máx foram feitas três mensurações consecutivas, com intervalo regular de um minuto. Para análise estatística dos dados, foram considerados os maiores valores obtidos nas três mensurações realizadas. Todos os indivíduos foram avaliados por um único observador, que utilizou método uniforme para administração da técnica.

Os participantes receberam esclarecimento sobre os procedimentos realizados durante a pesquisa e assinaram o termo de consentimento livre e esclarecido. 0 projeto de pesquisa foi aprovado pelo Comitê de Ética em Pesquisa (CEP) da USS e protocolado pelo Certificado de Apresentação para Apreciação Ética (CAAE), emitido pela Comissão Nacional de Ética em Pesquisa (CONEP) sob o registro CAAE - 0004.0.326.000-09.

As características antropométricas e clínicas dos grupos foram comparadas pelo teste da normalidade de Gaussen/Kolmogorov. A diferença entre os valores das medidas da avaliação da função ventilatória pulmonar pré e pós-posicionamento dos MMSS foi analisada através do GraphPad Prism versão 5.0, San Diego (CA, EUA), pelo teste $t$ Student pareado, e foram considerados significativos os valores de $p<0,05$.

\section{Resultados}

Os dados antropométricos e as variáveis clínicas do grupo $S$, com idade média de 38,67 $\pm 2,72$ anos e do grupo $H$, com idade média de 48,50 $\pm 4,80$ anos, estão expressos na Tabela 1 . Ambos os grupos apresentaram parâmetros antropométricos e clínicos semelhantes, sendo $71,09 \pm 1,87$, o peso dos indivíduos saudáveis, e o dos indivíduos hospitalizados, 61,13 $\pm 6,96 \mathrm{~kg}$. Os dois grupos passaram no teste de normalidade para todos os parâmetros.

Em relação à avaliação da função ventilatória pulmonar pré e pós-posicionamento MMSS, posicionados em abdução e rotação externa de ombro a $90^{\circ}$, associados à flexão de cotovelo a $90^{\circ}$ - assim como mostrado no Gráfico 1 do grupo S (Tabela 2) -, ela mostrou um aumento significativo do VM (Gráfico 1) e do VC (Gráfico 2) após o reposicionamento dos membros superiores, em que foram encontrados, respectivamente, os valores $\mathrm{p}<0,01$ e $\mathrm{p}=0,001$. 0 grupo $\mathrm{H}$ obteve $p=0,76$ para VM e 0,79 para VC.

Os outros parâmetros funcionais (FR, PFE, CVF, PE máx, PI máx) tiveram uma pequena queda após o reposicionamento dos membros superiores no grupo de indivíduos saudáveis.

\section{Discussão}

O padrão respiratório e o movimento toracoabdominal podem ser influenciados por vários fatores, como posicionamento do indivíduo, idade, sexo, sobrecarga respiratória, doenças neuromusculares, doenças pulmonares associadas ao aumento da resistência da via aérea e doença pulmonar obstrutiva crônica (DPOC) (9).

Em se tratando do posicionamento do indivíduo, alguns músculos, como trapézio, peitoral menor, escalenos e intercostais, que participam no posicionamento dos braços, podem ter funções posturais e ventilatórias. Assim, a simples elevação dos braços resulta em considerável aumento do consumo de oxigênio (16\%) e da ventilação pulmonar (24\%) em indivíduos normais (10).

As mudanças fisiológicas da função pulmonar no envelhecimento incluem mudanças em pulmões, caixa torácica, músculos respiratórios e movimentos respiratórios, caracterizados por redução da retração elástica dos pulmões, da complacência da parede torácica, da resposta a hipoxemia e hipercapnia e da força dos músculos respiratórios (11). Esta última, de acordo com alguns estudos, diminui aproximadamente $8-10 \%$ por década a partir dos 40 anos (12), e sua avaliação, através da pressão respiratória máxima, 
Tabela 1 - Dados antropométricos e variáveis clínicas do grupo de indivíduos saudáveis e do grupo de indivíduos hospitalizados

\begin{tabular}{lccc}
\hline Parâmetros & Grupo $\mathbf{S}$ & Grupo $\mathbf{H}$ & Teste de normalidade \\
\hline Idade $($ anos $)$ & $38,67 \pm 2,72$ & $48,50 \pm 4,80$ & + \\
Peso $(\mathrm{kg})$ & $71,09 \pm 1,87$ & $61,13 \pm 6,96$ & + \\
Altura $(\mathrm{m})$ & $1,66 \pm 0,01$ & $1,68 \pm 0,04$ & + \\
IMC $\left(\mathrm{Kg} / \mathrm{m}^{2}\right)$ & $25,66 \pm 0,76$ & $21,28 \pm 1,66$ & + \\
PAM $(\mathrm{mmHg})$ & $95,27 \pm 3,67$ & $98,68 \pm 2,71$ & + \\
FR (irpm) & $17,08 \pm 0,92$ & $14,75 \pm 1,31$ & + \\
FC (bpm) & $71,33 \pm 2,67$ & $70,00 \pm 5,71$ & + \\
\hline
\end{tabular}

Fonte: Dados da pesquisa.

Legenda: (+) indica que passou no teste da normalidade.

S: indivíduos saudáveis; $\mathrm{H}$ : indivíduos hospitalizados; IMC: índice de massa corporal; $\mathrm{Kg}$ : quilograma; m: metros; $\mathrm{Kg} / \mathrm{m}^{2}$ : quilograma-força por metro ao quadrado; PAM: pressão arterial média; mmHg: milímetros de mercúrio; FR: frequência respiratória; irpm: incursões respiratórias por minuto; FC: frequência cardíaca; bpm: batimentos por minuto.

Nota: os dados estão expressos em média \pm erro padrão da média.

Tabela 2 - Parâmetros funcionais mensurados no pré e no pós-posicionamento dos membros superiores, no grupo de indivíduos saudáveis

\begin{tabular}{cccc}
\hline Parâmetros funcionais & Pré & Pós & T student \\
\hline FR (irpm) & $17,33 \pm 1,42$ & $17,25 \pm 1,42$ & $\mathrm{p}=0,93$ \\
PFE $(/ /$ min) & $279,2 \pm 26,6$ & $269,6 \pm 16,3$ & $\mathrm{p}=0,65$ \\
CVF $(\mathrm{ml})$ & $1825 \pm 232,5$ & $1867 \pm 127,4$ & $\mathrm{p}=0,76$ \\
PE máx $\left(\mathrm{cmH}_{2} \mathrm{O}\right)$ & $90 \pm 6,88$ & $88,67 \pm 60,67$ & $\mathrm{p}=0,74$ \\
PI máx $\left(\mathrm{CmH}_{2} \mathrm{O}\right)$ & $-102 \pm 7,76$ & $-98 \pm 8,56$ & $\mathrm{p}=0,27$ \\
\hline
\end{tabular}

Fonte: Dados da pesquisa.

Legenda: São considerados estatisticamente significativos os valores de $p<0,05$.

FR: frequência respiratória; irpm: incursões respiratórias por minuto; PFE: pico de fluxo expiratório; //min: litros por minuto; CVF: capacidade vital forçada; PE máx: pressão expiratória máxima; $\mathrm{cmH2O}$ : centímetros de água; PI máx: pressão inspiratória máxima.

Nota: Os dados estão expressos em média \pm erro padrão da média.

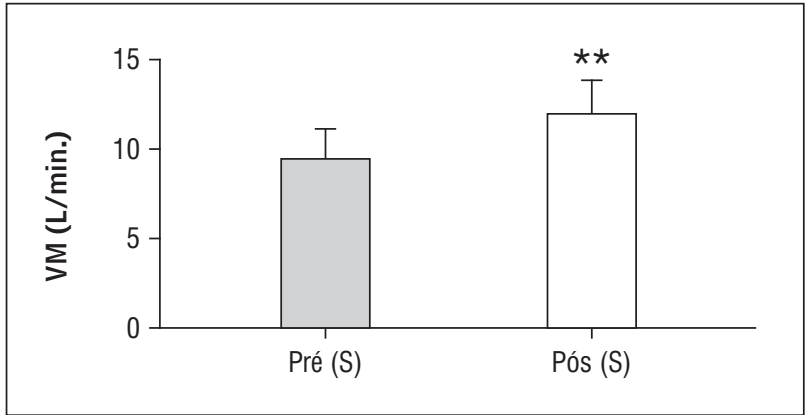

Gráfico 1 - Volume minuto (VM) no pré e no pós-posicionamento dos MMSS do grupo de indivíduos saudáveis $(\mathrm{S}) ; \mathrm{p}<0,01 ; \mathrm{n}(\mathrm{S})=12$

Fonte: Dados da pesquisa.

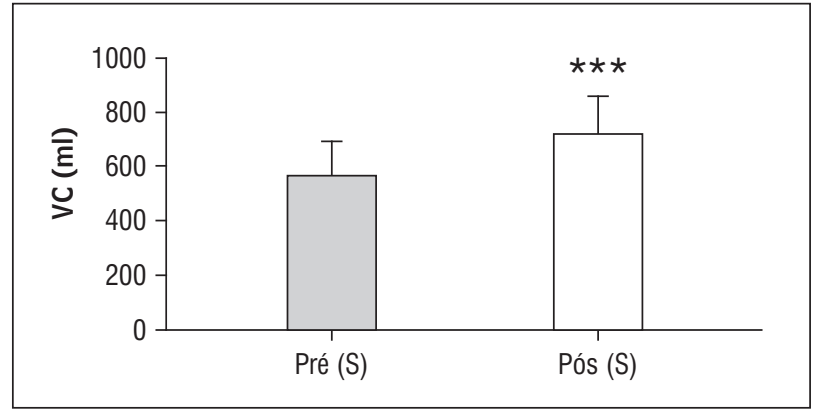

Gráfico 2 - Volume corrente (VC) no pré e no pós-posicionamento dos MMSS do grupo de indivíduos saudáveis $(S) ; p=0,00 ; n(S)=12$

Fonte: Dados da pesquisa. 
tem sido usada por sua importância no diagnóstico e no prognóstico de doenças neuromusculares, pulmonares e cardiovasculares (13).

As doenças respiratórias ocupam a segunda posição entre as doenças que acarretam os maiores custos com internações hospitalares pelo Sistema Único de Saúde (SUS). Atelectasias, hipoxemia, pneumonia e derrame pleural são, inclusive, as principais complicações no pós-operatório de pacientes submetidos a cirurgia abdominal alta ou torácica. Essas complicações podem levar o indivíduo a apresentar um padrão de movimento toracoabdominal ineficaz, alterando a distribuição regional da ventilação, e, assim, aumentar o risco de morbidade e de mortalidade, prolongando os dias de hospitalização e elevando os custos na atenção à saúde desses indivíduos (14). Em 2003, foram gastos, aproximadamente, 660 milhões de reais. Ao considerar as internações de pessoas com mais de 40 anos de idade, a DPOC, especificamente, passa a ocupar o segundo lugar. DPOC é considerada a sétima maior causa de morte no Brasil e a quarta no mundo. Estimase que os pacientes mais graves cheguem a representar um custo anual de 2.911 dólares, os moderados, 2.047 dólares, e os leves, 1.484 dólares $(15,16)$.

Complicações respiratórias podem ser minimizadas ou evitadas com a utilização de protocolos de atendimento de fisioterapia respiratória. Vários métodos têm sido utilizados, tais como respiração com pressão positiva intermitente, exercícios com respiração profunda, inspirometria de incentivo e fisioterapia torácica convencional $(17,18)$. As técnicas reexpansivas utilizadas na reabilitação da função ventilatória pulmonar estabelecem, primariamente, 0 posicionamento do corpo: é um meio de intervenção capaz de colocar em vantagem os músculos da bomba ventilatória. Isso porque a relação comprimento-tensão muscular é beneficiada, gerando vantagem biomecânica eficaz para promover maior força de contração. Dessa forma, o posicionamento dos membros superiores (MMSS), visando ao alongamento das fibras do músculo peitoral maior, contribui quantitativamente na mecânica ventilatória pulmonar (19).

Uma boa estratégia deve, ainda, levar em consideração a monitorização clínica baseada na minimização de possíveis fatores que possam interferir na análise da função pulmonar, como o tipo de aparelho utilizado, o modo de execução, a temperatura ambiente e a altitude. Essas variáveis são atenuadas com a utilização de equipamentos calibrados e com acurácia técnica para execução do exame (20).
Ao se tratar de pesquisas envolvendo seres humanos, a heterogeneidade da amostra também é um fator importante que deve ser controlado. Indivíduos obesos, por exemplo, apresentam diminuição dos volumes pulmonares, bem como da complacência tóracopulmonar, pois a resistência imposta aos músculos inspiratórios aumenta a impedância do influxo de ar para as vias aéreas. No presente estudo, as análises das variáveis antropométricas, como peso, altura e IMC, demonstraram a característica eutrófica dos indivíduos categorizados como saudáveis e hospitalizados $(21,22)$.

A geração de força muscular depende do comprimento da fibra e de sua velocidade de encurtamento, do número de unidades contráteis ativadas e da frequência do disparo do neurônio motor. Foi observado que apenas o posicionamento dos MMSS não foi capaz de alterar a PE máx e a PI máx. Entretanto, foi demonstrado, em outro estudo, que o alongamento dos músculos inspiratórios melhorou a PE máx, a PI máx e o VC dos indivíduos que foram submetidos a um programa de alongamento de longo prazo. É provável que somente o posicionamento não é capaz de ativar um maior número de fibras contráteis (23).

Nos homens, sabe-se que há predominância da expansão das regiões pulmonares inferiores, caracterizando o padrão ventilatório costoabdominal. Neste estudo, foi considerado que o posicionamento dos MMSS em 90 de abdução e rotação externa promoveria maior expansão das regiões superiores do tórax, sendo responsável pelo aumento de VM e VC. Essa hipótese foi confirmada, já que o reposicionamento dos MMSS promoveu maior expansão da parte superior do tórax e, consequentemente, aumentou o VM e o VC. Isso porque, quando o músculo ventilatório é posicionado em vantagem biomecânica, a função ventilatória pulmonar pode ser beneficiada (24-26).

Foi demonstrado que os valores de CVF e PFE estiveram abaixo do previsto fisiologicamente para os indivíduos adultos. Especula-se que esse achado esteja relacionado com a posição habitualmente utilizada para realização dos testes de função pulmonar, em postura ortostática ou sentada. Os valores obtidos em decúbito dorsal, como nesse caso, podem ser diminuídos em $25 \%$ ou mais em relação aos obtidos na posição sentada (27-29).

A medida da função pulmonar deve ser obtida durante a avaliação clínica, visando quantificar a gravidade de uma doença já diagnosticada, além de avaliar os riscos de procedimentos cirúrgicos. Dessa forma, 
é possível uma intervenção clínica antes da instalação de danos pulmonares irreversíveis, prevenindo a morbidade e a mortalidade precoce, uma vez que as mudanças aceleradas existentes na função pulmonar estão intimamente relacionadas com a diminuição da sobrevida (30).

\section{Conclusões}

O posicionamento dos MMSS com o ombro em $90^{\circ}$ de abdução e rotação externa, associado à flexão de cotovelo a $90^{\circ}$, melhorou o VM e o VC em indivíduos saudáveis, sugerindo que a adoção desse posicionamento previamente à realização de técnicas de fisioterapia respiratória é um recurso para o aumento do volume minuto e do volume corrente.

\section{Referências}

1. Sarro KJ. Proposição, validação e aplicação de um novo método para a análise cinemática tridimensional da movimentação da caixa torácica durante a respiração [tese]. Campinas: Universidade Estadual de Campinas; 2007.

2. Silva LCC, Rubin AS, Silva LMC, Fernandes JC. Espirometria na prática médica. Rev AMRIGS. 2005;49(3):183-94.

3. Paisani DM, Chiavegato LD, Faresin SM. Volumes, capacidades pulmonares e força muscular respiratória no pós-operatório de gastroplastia. J Bras Pneumol. 2005;31(2):125-32.

4. Castro HA. Efeitos da poluição do ar na função respiratória de escolares. Rev Saúde Pública. 2009;43(1):26-34. PMid:19169573.

5. Pereira CAC, Neder JA. Diretrizes para testes de função pulmonar. J Bras Pneumol. 2002;28(Supl 3):1-80.

6. Fiore JF Jr, Paisani DM, Franceschini J, Chiavegato LD, Faresin SM. Pressões respiratórias máximas e capacidade vital: comparação entre avaliações através de bocal e de máscara facial. J Bras Pneumol. 2004;30(6):515-20.

7. Garcia E, Silvério AO, Brandão AB, Moreira JS. Função pulmonar na cirrose hepática. J Bras Pneumol. 1997;23(5):225-30.

8. Melo MH. Efeitos da gastroplastia redutora sobre a função pulmonar à beira do leito no pós-operatório de mulheres obesas [dissertação]. Brasília: Universidade Católica de Brasília; 2006.
9. Parreira VF, Bueno CJ, França DC, Vieira DSR, Pereira DR, Britto RR. Padrão respiratório e movimento toracoabdominal em indivíduos saudáveis: influência da idade e do sexo. Rev Bras Fisioter. 2010;14(5):411-6. doi:10.1590/ S1413-35552010000500010.

10. Velloso M, Jardim JR. Funcionalidade do paciente com doença pulmonar obstrutiva crônica e técnicas de conservação de energia. J Bras Pneumol. 2006; 32(6):580-6. doi:10.1590/S1806-37132006000600017.

11. Britto RR, Zampa CC, Oliveira TA, Prado LF, Parreira VF. Effects of the aging process on respiratory function. Gerontology. 2009;55:505-10. doi:10.1159/000235853.

12. Costa D, Gonçalves HA, Lima LP, Ike D, Cancelliero KM, Montebelo MIL. Novos valores de referência para pressões respiratórias máximas na população brasileira. J Bras Peneumol. 2010;36(3):306-12. doi:10.1590/ S1806-37132010000300007.

13. Simões RP, Deus APL, Auad MA, Dionísio J, Mazzonetto $\mathrm{M}$, Borghi-Silva A. Maximal respiratory pressure in healthy 20 to 89 year-old sedentary individual of central. Rev Bras Fisioter. 2010;14(1):60-7. doi:10.1590/ S1413-35552010000100010.

14. Yamaguti WPS, Sakamoto ET, Panazzolo D, Peixoto CC, Cerri GG, Albuquerque ALP. Mobilidade diafragmática durante espirometria de incentivo orientada a fluxo e a volume em indivíduos sadios. J Bras Peneumol. 2010;36(6):73845. doi:10.1590/S1806-37132010000600011.

15. Miravitles M. Avaliação econômica da doença pulmonar obstrutiva crônica e de suas agudizações. J Bras Pneumol. 2004;30(3):274-85.

16. Godoy DV. Doenças respiratórias como causa de internações hospitalares de pacientes do Sistema Único de Saúde num serviço terciário de clínica médica na região nordeste do Rio Grande do Sul. J Bras Pneumol. 2001;27(4):193-8.

17. Dias CM, Plácido TR, Ferreira MFB, Guimarães FS, Menezes SLS. Inspirometria de incentivo e breath stacking: repercussões sobre a capacidade inspiratória em indivíduos submetidos à cirurgia abdominal. Rev Bras Fisioter. 2008;12(2):94-9.

18. Alves LA, Brunetto AF. Adaptação do threshold IMT para testes de resistência dos músculos inspiratórios. Rev Bras Fisioter. 2006;10(1):105-12. doi:10.1590/ S1413-35552006000100014.

19. Dias CS. Cinemática da cintura escapular, coluna cervical e torácica em idosos com doença pulmonar. obstrutiva crônica [dissertação]. Belo Horizonte: Universidade Federal de Minas Gerais; 2007. 
20. Terra Filho J. Avaliação laboratorial da função pulmonar. Medicina. 1998;31:191-207.

21. Helene A Jr, Saad R Jr, Stirbulov R. Avaliação da função respiratória em indivíduos submetidos à abdominoplastia. Rev Col Bras Cir. 2003;33(1):45-50.

22. Caséca M, Andrade L, Britto M. Avaliação da função pulmonar em crianças e adolescentes no pré e pós-operatório de correção cirúrgica de valvulopatia reumática. J Pediatr. 2006;82(2): 144-50.

23. Cunha APN. Efeito do alongamento sobre a atividade dos músculos inspiratórios na DPOC. Saúde Rev. 2005;7(17):13-9.

24. Menezes V, Leal RB, Moura MM, Granville-Garcia AF. Influência de fatores socioeconômicos e demográficos no padrão de respiração: um estudo piloto. Rev Bras Otorrinolaringol. 2007;73(6):826-34. doi:10.1590/ S0034-72992007000600014.

25. Pinheiro CHJ. Modificação do padrão respiratório melhora o controle cardiovascular na hipertensão essencial. Arq Bras Cardiol. 2007;88(6):651-9. doi:10.1590/ S0066-782X2007000600005.

26. Emmerich JC. Monitorização respiratória: fundamentos. 2a ed. Rio de Janeiro: Revinter; 2001.
27. Duarte AA, Pereira CA, Rodrigues SCS. Validação de novos valores previstos brasileiros para espirometria forçada na raça branca e comparação com os valores previstos obtidos por outras equações de referência. J Bras Pneumol. 2007;33(5):527-35. PMid:18026650.

28. Parreira V, França DC, Zampa CC, Fonseca MM, Tomich GM, Britto RRI. Pressões respiratórias máximas: valores encontrados e preditos em indivíduos saudáveis. Rev Bras Fisioter. 2007;11(5):361-8. doi:10.1590/ S1413-35552007000500006.

29. Badr C, Elkins MR, Ellis ER. The effect of body position on maximal expiratory pressure and flow. Aust J Physiother. 2002;48:95-102. PMid:12047207.

30. Faria AG. Avaliação da função pulmonar e da mecânica respiratória em adolescentes obesos [dissertação]. Campinas: Universidade Estadual de Campinas; 2004.

Recebido: $12 / 02 / 2011$

Received: 02/12/2011

Aprovado: $13 / 08 / 2012$

Approved: 08/13/2012 